\title{
Experimental and numerical polar scans of several anisotropic materials using pulsed and harmonic ultrasonic beams
}

\author{
M. Kersemans, I. De Baere, W. Van Paepegem \& J. Degrieck \\ Department of Materials Science and Engineering, Ghent University, Technology park 903, 9052 Zwijnaarde, \\ Belgium \\ F. Zastavnik, J. Gu \& H. Sol \\ Department Mechanics of Materials and Constructions, Free University of Brussels, Pleinlaan 2, 1050 \\ Brussels, Belgium
}

\begin{abstract}
Ultrasonic non-destructive testing is a well known technique in present days, in which the Cscan is the most wide spread. Though, because of the inherent limitations of most methods to quantitatively characterize (damaged) composite materials, the quest for more sophisticated methods is put forward. This study reports experimentally registered polar scans using an in house developed ultrasonic test setup for some typical composite materials. Both pulsed and harmonic ultrasonic beams are considered, which impinge onto the immersed anisotropic layer under investigation. Numerical computations are shown and compared with the experimental results. The experimental polar scan of a carbon fabric/PPS laminate with an artificially added delamination shows a drastic change in the observed patterns, compared to the one of the undamaged carbon fabric/PPS laminate. Combined with the sensitivity to the local stiffness tensor, a polar scan can become a great tool to quantitatively evaluate (damaged) composite materials.
\end{abstract}

\section{INTRODUCTION}

Due to the increasing use of composite materials in critical applications demanding light weight to high strength, reliable evaluation tools are necessary. Within these tools, the classical ultrasonic C-scan has already proven its usefulness in qualitatively visualizing defects, delaminations, etc. (Balasubramaniam and Whitney, 1996, Amenabar I., 2011). A so-called polar scan (Vandreumel and Speijer, 1981), which makes use of ultrasound from all possible angles of oblique incidence, can serve as a prominent successor of the more classical methods. By gathering the reflected (or transmitted) amplitude of the ultrasonic beam, one is able to build a polar plot which covers a certain solid angle.

This paper reports experimentally registered polar scans which are compared with numerically computed polar scans for a variety of composite materials, using both pulsed and harmonic ultrasonic beams. Typically, dark rings are observed in a polar plot, which are linked to the generation of critical bulk waves (pulsed regime) or to the generation of guided waves (harmonic regime) (Declercq et al., 2006a, Declercq et al., 2006b, Degrieck et al., 2003). Both types of waves are vigorously entangled with the elastic constants of the material (Rose, 1999, Nayfeh and Chimenti, 1988, Auld, 1990). This im- plicates that a polar scan is a great candidate tool to investigate fatigue of anisotropic materials nondestructively, where the mapped amplitudes are a local fingerprint of the investigated material (Declercq et al., 2004, Degrieck et al., 2003). Indeed, many composites show a gradual degradation of their elastic properties, when subjected to fatigue loading. Furthermore, an artificially delaminated carbon fabric/PPS laminate is investigated with a harmonic ultrasonic beam. Severe changes are observed in the 'fingerprint', which implies that the technique can efficiently detect delaminations. Results are shown for the virginal and delaminated sample.

\section{PHYSICAL BACKGROUND OF POLAR SCAN}

According to Christoffel's equation, an unbounded material possesses three distinct bulk waves, one (quasi-) longitudinally and two (quasi-) transversally polarized ones. Each of them having a characteristic phase velocity $c_{p h}$ (analogously a characteristic wave vector $\boldsymbol{k}$ ), which is determined by the physical properties, more precise the elasticity constants $C_{i j}$ and density $\rho$, of the material under investigation (Auld, 1990, Rose, 1999). To promote the transfer of acoustical energy in the solid layer, a coupling medium, 
e.g water, is often used. Snell-Descartes' law which states that $k_{1}=k_{1}^{l i q}$ and $k_{2}=k_{2}^{l i q}$, for interfaces perpendicular to $k_{3}$, must be taken into account for external borne (denoted with superscript 'liq') sound propagating in a material. This leads to the concept of critical angles, at which the impinging wave is refracted with its wave vector $\boldsymbol{k}$ parallel to the interface. A sharp discontinuity is observed in the reflection (transmission) curves (Rose, 1999, Auld, 1990), leading to the characteristic patterns of a polar plot. The critical bulk angles, computed for some typical composite materials, immersed in water, are presented in polar form in Figure 1.
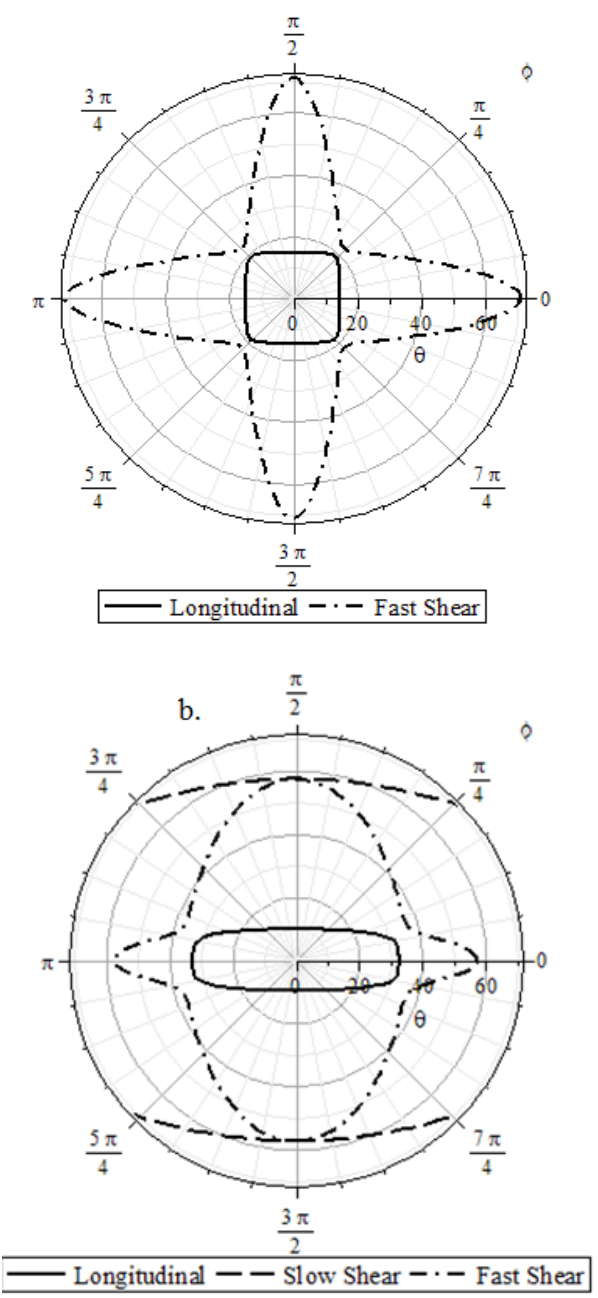

Figure 1. Critical bulk angles for (a) carbon fabric/PPS laminate and (b) unidirectional carbon/epoxy. The anisotropic nature of the materials clearly being reflected in the curves. The slow shear wave is absent in (a), partially absent in (b).

The calculations for the orthotropic carbon fabric/PPS laminate are performed using the material parameters given in (Daggumati et al., 2010), while the constants for UD carbon/epoxy are determined within our research team. Both set of parameters are listed in Table 1.
Table 1: Material constants of UD carbon/epoxy (65\%-35\% proportion) and carbon fabric/PPS laminate used for computations.

\begin{tabular}{|c|c|c|}
\hline & $\begin{array}{l}\text { UD Carbon } \\
\text { epoxy }\end{array}$ & $\begin{array}{c}\text { Carbon fabric/PPS } \\
\text { laminate }\end{array}$ \\
\hline$E_{11}[\mathrm{GPa}]$ & 154.13 & 56.49 \\
\hline$E_{22}[G P a]$ & $\begin{array}{r}14.79 \\
-\cdots . . .\end{array}$ & 56.41 \\
\hline$E_{33}[G P a]$ & $\begin{array}{r}14.79 \\
-\cdot . \cdots\end{array}$ & 10.53 \\
\hline $\mathbf{v}_{12}$. & 0.17 & 0.08 \\
\hline & $\begin{array}{l}0.17 \\
\cdots . . .\end{array}$ & 0.41 \\
\hline$v_{23}$ & $\begin{array}{l}0.30 \\
0 . .\end{array}$ & 0.41 \\
\hline $\mathrm{G}_{12}[\mathrm{MPa}]$ & $\begin{array}{c}7480 \\
\cdots\end{array}$ & 4280 \\
\hline $\mathrm{G}_{13}[\mathrm{MPa}]$ & $\begin{array}{c}7480 \\
\cdots-. .\end{array}$ & 3048 \\
\hline$G_{23}[\mathrm{MPa}]$ & 3370 & 3045 \\
\hline$\rho \quad\left[K g / m^{3}\right]$ & 1614 & 1750 \\
\hline
\end{tabular}

One notices the (partial) absence of the slow shear wave in Figure 1, which is simply caused by the fact that its associated phase velocity $c_{\mathrm{ph}}$ is smaller than the longitudinal phase velocity of water. According to Snell-Descartes' law, this would lead to an imaginary refractive angle for the bulk waves, which is physically impossible.

In reality, structural materials are bounded in space, resulting to the possible generation of so called (quasi-) Lamb waves (I.A.Viktorov, 1967). These waves propagate along the plate structure, with a standing wave pattern through the thickness of the layer. Because they are physically guided by the plate, the Lamb waves are often termed as 'guided modes'. Mathematically, the guided modes can be understood as a linear combination of the bulk waves, having a resultant wave vector $\boldsymbol{k}$ along the interface (Auld, 1990). Contrary to the case of bulk waves, the number of possible Lamb modes is not limited to a fixed number. Acoustical frequency $f$ and thickness $d$ of the material largely prescribe the number of Lamb modes, while the material parameters $\left(C_{i j}\right.$ and $\left.\rho\right)$ define their exact conditions of existence, i.e. the needed incident angle to efficiently generate them. Similar to the case of bulk waves, the generation of a Lamb mode results in a peak (valley) in the topology of the reflection (transmission) coefficient (Nayfeh and Chimenti, 1988). For completeness, it is mentioned that material damping (which can be effectively modeled by considering an imaginary elasticity tensor), does not affect the position of the pattern, but rather the amplitude of the polar plot. In Figure 2, several curves are observed, prescribing the existence conditions of Lamb waves for a carbon fabric/PPS laminate in different $\varphi$-directions. The frequency-thickness product $f d$ is put on the x-axis, while the $y$-axis is characterized by the incident an- 
gle $\theta$. Note the addition of the bulk solutions in Figure 2 (indicated with arrows), which are recognized by their non-dispersive course. The anisotropic nature of the considered material, introduces the dependency of polar angle $\varphi$ on the generation of Lamb waves. Along the symmetry axes, the term 'quasi', which is a consequence of the anisotropic material properties, can be omitted, thus resulting in 'pure' modes.
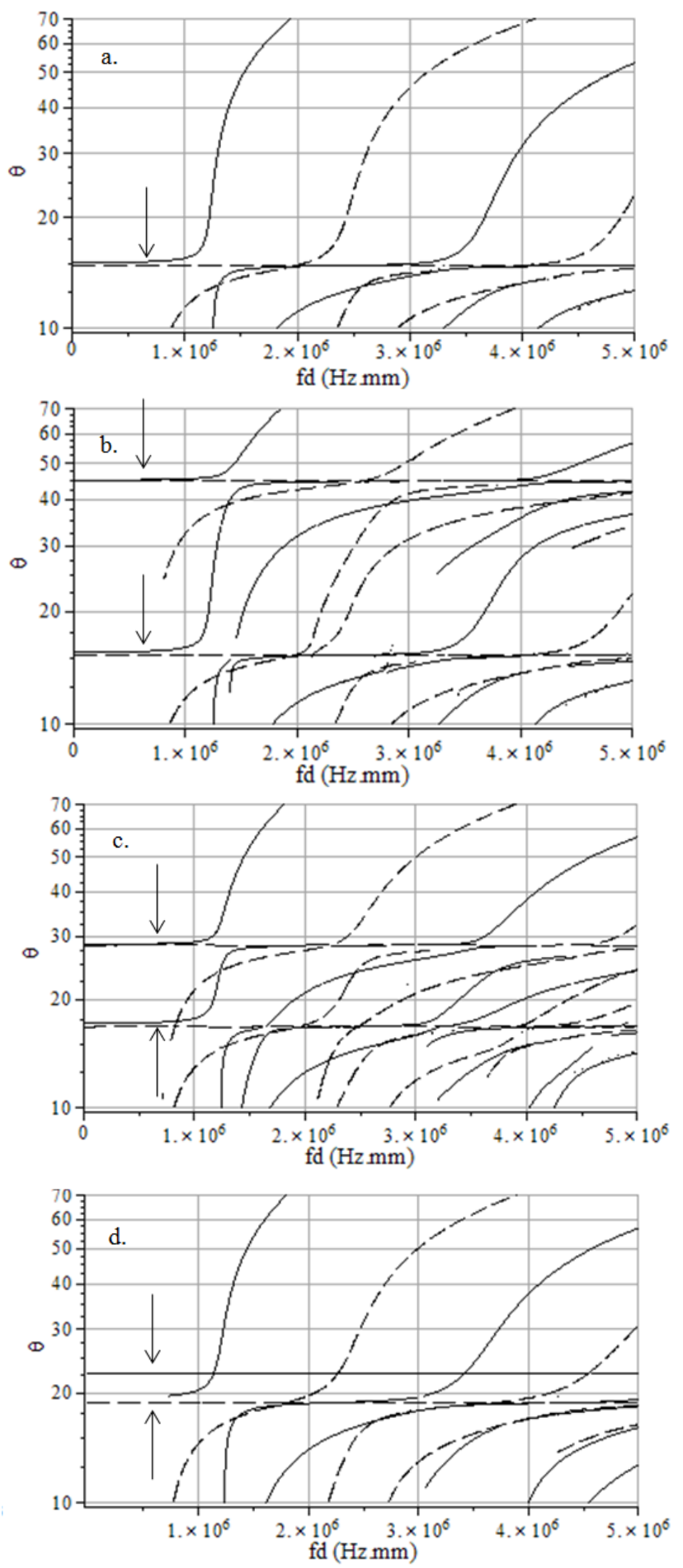

Figure 2. Dispersion curves $\theta$ (fd) for an immersed carbon fabric/PPS laminate for several polar angles: $\varphi=0^{\circ}$ (a), $\varphi=15^{\circ}$ (b), $\varphi=30^{\circ}$ (c) and $\varphi=45^{\circ}$ (d).
In practice, a pulsed or harmonic incident ultrasonic beam can be used. It is observed that a pulsed beam (typical values for $f d$ are in the range of 2$10 \mathrm{MHz} . \mathrm{mm}$ ) produces a fingerprint which converges to the patterns associated with the bulk solutions. This can be understood in terms of the frequency content of a pulsed beam, together with the highly dispersive character of the Lamb modes. The (local) convergence of the Lamb modes towards the bulk solutions does the remains. An incoming harmonic beam on the other hand, produces the conditions to efficiently excite a Lamb mode. Hence the fingerprint depends upon the frequency $f$ of the considered harmonic source (Declercq et al., 2006a, Degrieck et al., 2003).

\section{EXPERIMENTAL SETUP}

To perform the measurements with good accuracy, an in-house developed robot is constructed (Figure 3). The setup consists of 5 axes of freedom, which allows the operator to perform both a C-scan and a polar scan of the material under investigation. The steering of the axes is programmed using LABVIEW in order to describe part of a spherical surface with its center point located at the zone under investigation. Position feedback is assured by linear respectively rotary encoders with an accuracy of $0.007 \mathrm{~mm}$ respectively $0.1^{\circ}$.

The machine can be operated in both the pulsed regime, using the OMNISCAN pulse generator (center frequency of $1-5 \mathrm{MHz}$ ), and the harmonic regime, using the GYMNA 330 apparatus operating at $f=1 \mathrm{MHz}$. The coupling medium is water, enhancing the energy transfer into the solid layer. The measurements in this report are gathered in transmission, although it could as easily be done in reflection. Using an acoustic mirror at the backside, one is able to operate in the double-transmission regime, which shows considerably more details because the acoustic wave undergoes two times the influence of the material. However, practical considerations prevent this technique to be employed in future industrial applications and thus it is not further investigated. We used the Gen5ioscilloscope as well as the USIP20 to process the large amount of data. In order to further reduce the experimental time, the symmetry of the material can be exploited. 


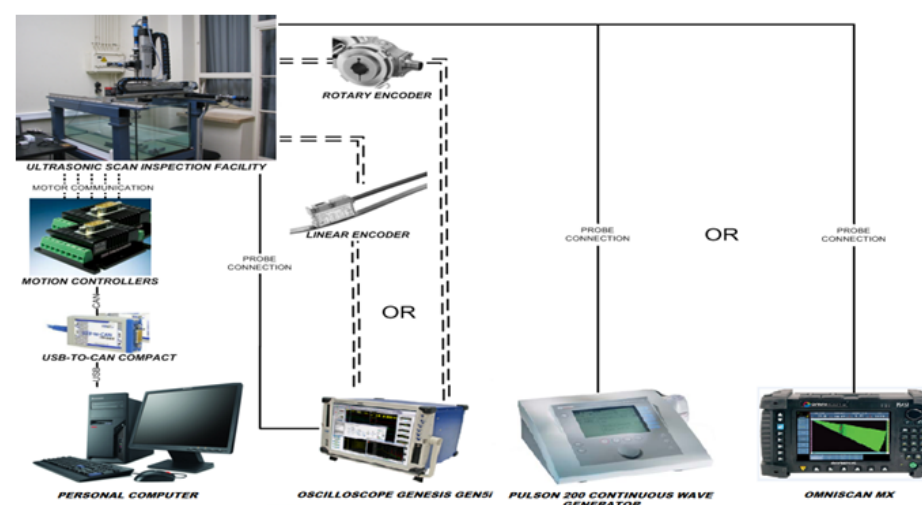

Figure 3. Experimental setup in which the GEN5i is used for data-acquisition.

\section{RESULTS AND DISCUSSION}

Figure 4 shows numerically computed polar scans in transmission for the carbon fabric/PPS laminate (thickness $d=2 \mathrm{~mm}$ ) for several operating frequencies. For clarity, the numerical and experimental results are normalized in this report. Comparison of the computed polar plots with the dispersion curves (Figure 2), clearly shows the link between the observed dark regions and the generation of Lamb modes. For high $f d$-values, the Lamb solutions converge towards the bulk solutions, causing the dark regions being pushed outwards to the bulk solutions. This behaviour is highlighted in Figure 4d, by superimposing the critical bulk angles onto the polar plot.
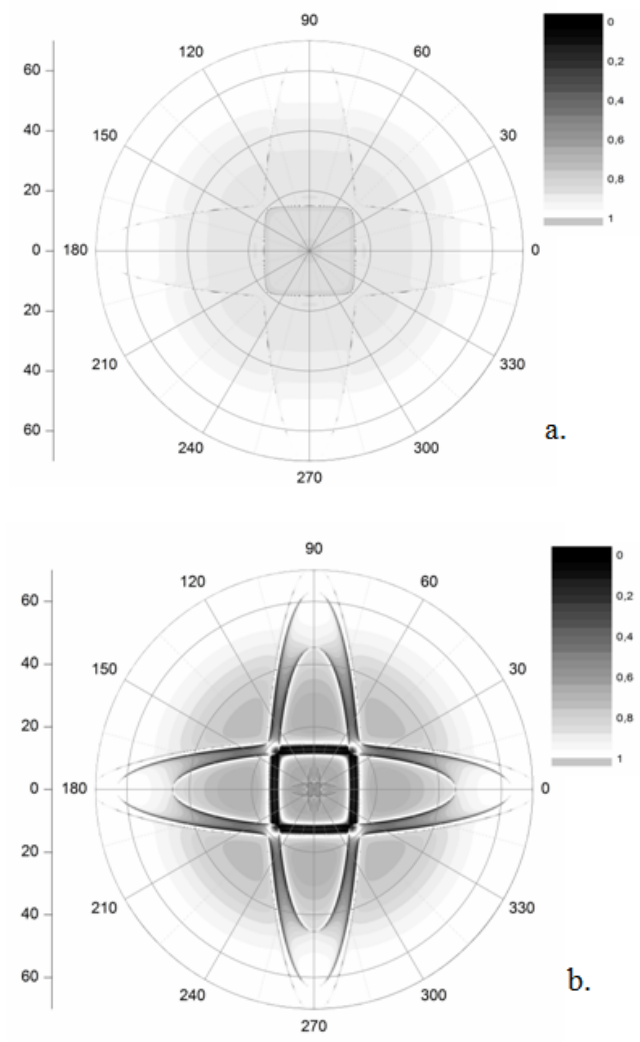
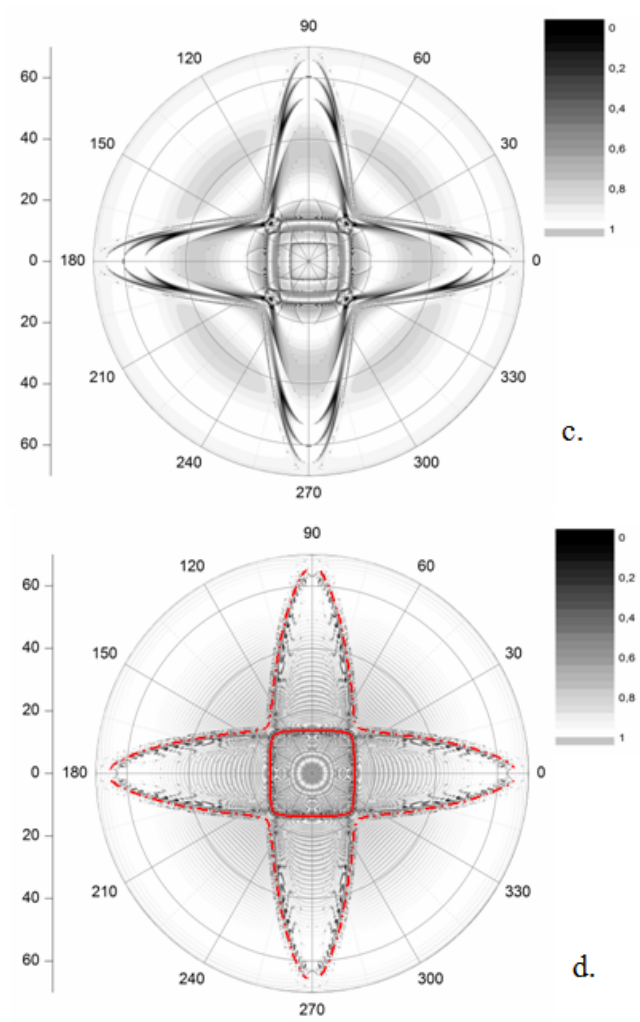

Figure 4. Numerically computed polar scans in transmission of an immersed carbon fabric/PPS laminate, with thickness $\mathrm{d}=2$ $\mathrm{mm}$, using an incident harmonic beam: $\mathrm{fd}=0.2 \mathrm{MHz} . \mathrm{mm}(\mathrm{a})$, $\mathrm{fd}=2 \mathrm{MHz} \cdot \mathrm{mm}$ (b), fd = $5 \mathrm{MHz} \cdot \mathrm{mm}$ (c) and fd = $25 \mathrm{MHz} \cdot \mathrm{mm}$ with the contour of the critical bulk angles superimposed (d).

Some preliminary experiments of the carbon fabric/PPS laminate are performed using a harmonic ultrasonic beam with frequency $f=1 \mathrm{MHz}$ (Figure 5). The assumption that the carbon fabric/PPS laminate behaves as an orthotropic material, is roughly reflected in the observed experimental fingerprints for both samples. The experimental data of sample 1 (thickness $d \approx 2 \mathrm{~mm}$ ) show some peculiarities (Figure 5a):

1. the shape of the 'arms' slightly differs for equivalent polar angles

2. the intensity of the 'arms' slightly differs for equivalent polar angles.

Close inspection of the 'arms' reveals the appearance of two dark contours (indicated with arrows for one arm), similar as seen in the numerically computed polar plot in Figure $4 \mathrm{~b}$. For the second sample in Figure $5 \mathrm{~b}$ (thickness $d \approx 6 \mathrm{~mm}$ ), one observes darker and broadened contours of the narrowed 'arms'. Furthermore, a contour is observed which seems to be quasi-circular, thus reflecting isotropic behaviour, at $\theta=40^{\circ}$ (indicated with an arrow). Figure $4 \mathrm{c}$ shows a similar sort of pattern in the numerically calculated plot. Indeed, for a vertically polarized shear wave, the plane containing the depth axis, can be regarded as an isotropic plane for the mentioned material. The inner square of the polar plot of sample 2 is largely 
unaltered compared to the one of sample 1 , which is easily explained using the dispersion curves (Figure 2).
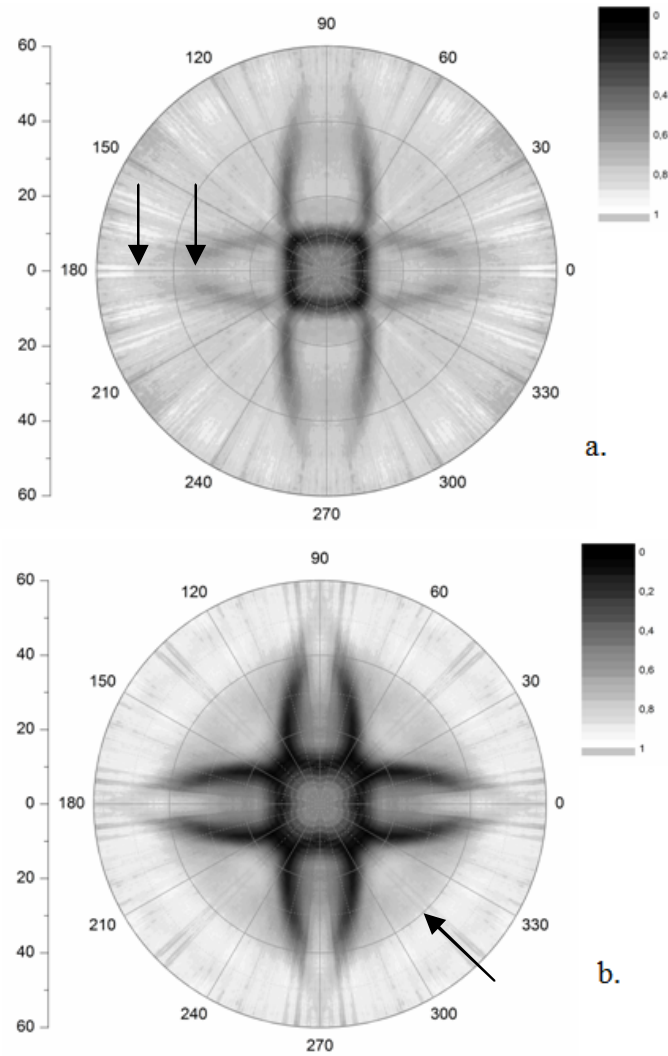

Figure 5. Experimentally registered polar scans in transmission of an immersed carbon fabric/PPS laminate, using an incident harmonic beam of $1 \mathrm{MHz}$ : $\mathrm{fd} \approx 2 \mathrm{MHz} \cdot \mathrm{mm}$ (a) and $\mathrm{fd} \approx 6$ MHz.mm (b). Some indicators are added for clarity.

Results for UD carbon/epoxy with thickness $d \approx 2 \mathrm{~mm}$ using a pulsed beam with center frequency $f=1 \mathrm{MHz}$, thus resulting in a value of $f d \approx 2 \mathrm{MHz} . \mathrm{mm}$, are presented in Figure 6. One unambiguously recognizes the experimental fingerprint within the numerical results, although it is rigidly rotated over $\sim 23^{\circ}$ (the symmetry plane of the sample was not aligned with the starting scan direction). The degeneracy of the two shear bulk waves, introduced by the equivalence of the 12-direction and the 13direction, is clearly seen.

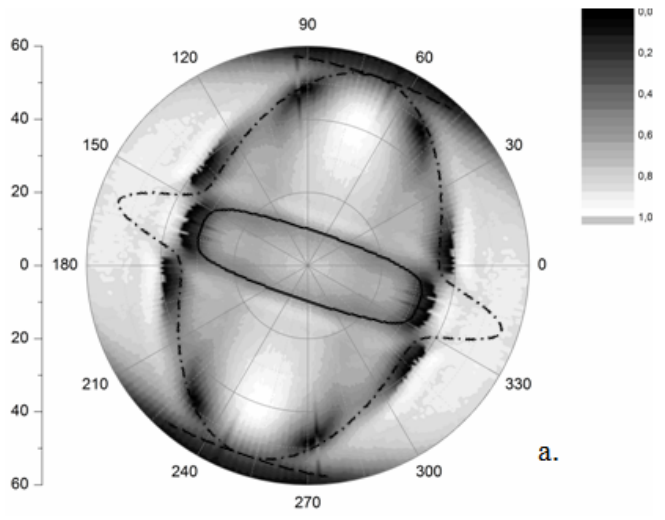

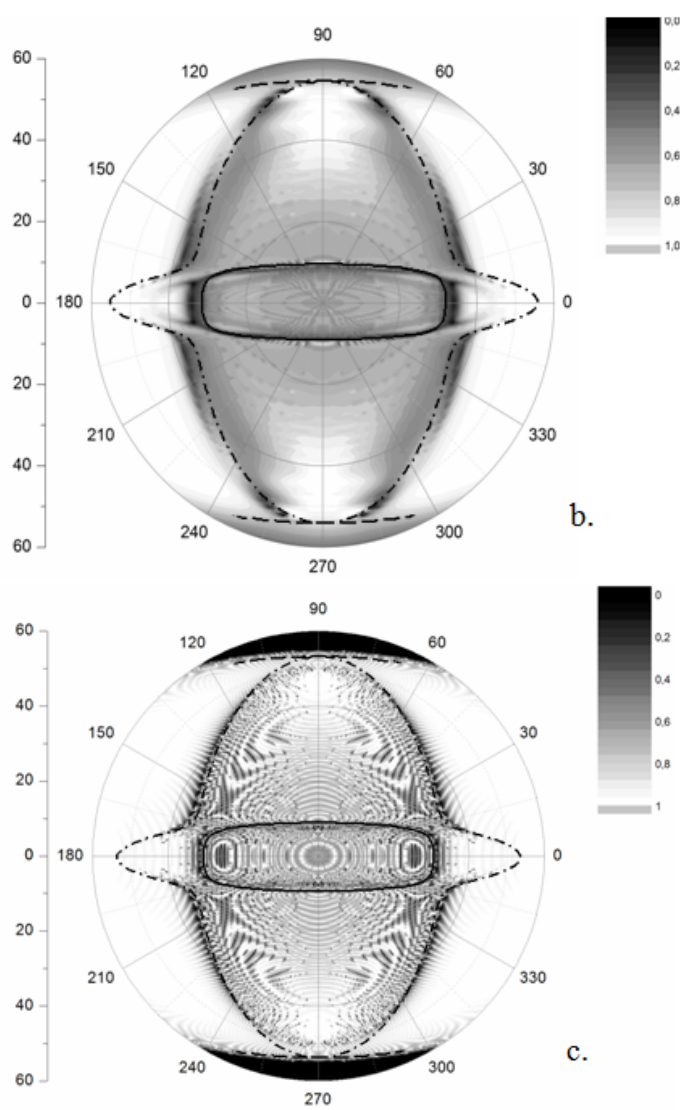

Figure 6. Experimentally registered (a) and numerically computed (b) polar scan in transmission of UD carbon/epoxy (thickness $\mathrm{d} \approx 2 \mathrm{~mm}$ ) for a pulsed ultrasonic beam with center frequency $\mathrm{f}=1 \mathrm{MHz}$. Numerically calculated polar scan using a harmonic beam at high fd-value (c). The critical bulk angles are superimposed on the shown polar plots.

Figure 7 shows the experimentally recorded polar scan for a delaminated carbon fabric/PPS laminate, with thickness $d \approx 6 \mathrm{~mm}$, using a harmonic beam operating at frequency $f=1 \mathrm{MHz}$. Compared with the results of the virginal carbon fabric/PPS laminate (Figure 5b), the overall contours are recognized up to some extent. Though, one unambiguously notes the change in patterns which goes with the delamination. Several scans are performed on delaminated composite materials, all showing considerable changes in its characteristic fingerprint. Overall lower amplitudes are noticed, compared to the fingerprint of the undamaged sample. This darkening effect is due to the presence of the artificial delamination, which hinders the acoustical energy to efficiently penetrate through the material. 


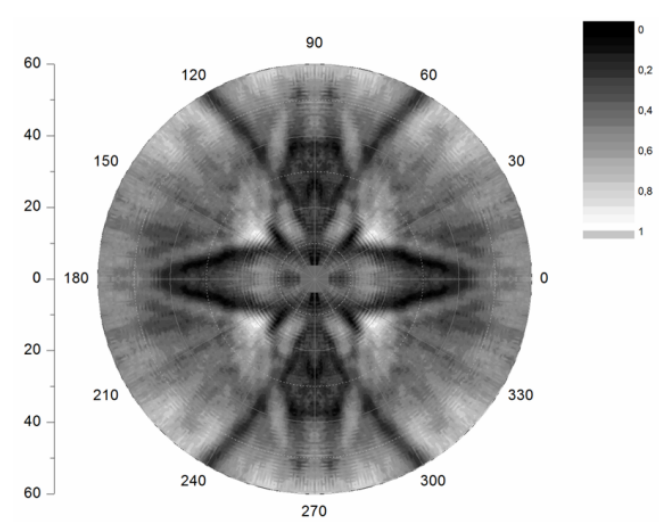

Figure 7. Experimentally registered polar scan in transmission of an immersed carbon fabric/PPS laminate, with frequencythickness $\mathrm{fd} \approx 6 \mathrm{MHz}$. mm, with an artificially added delamination.

In the near future, the experimental setup will be refined in order to enhance the contrast and to lower the experimental time. Emphasis will be put in the inverse modeling to retrieve the exact material constants. This will be of uttermost importance to assess possible fatigue damage.

\section{CONCLUSION}

We presented numerically computed and experimentally recorded polar scans, using harmonic and pulsed ultrasound, for two typical anisotropic materials immersed in water. Several interesting phenomena are recognized, linking the mapped amplitude to the internal stiffness of the investigated material. Overall, there is good agreement between the experiments and theory. Furthermore, it is shown that the characteristic fingerprint is very sensitive to delaminations, which is regarded as a major failure mechanism, besides fatigue, in typical structural applications.

The fact that the reported method (i) is easy to implement in industrial application, (ii) gathers information locally, (iii) can be simulated easily, (iv) works in reflection/transmission (v) shows great sensitivity to delaminations and fatigue and (vi) provides information about the phase- as well as the group velocities, makes it an interesting candidate in the field of quantitative non-destructive testing of complex structural components.

\section{REFERENCES}

AMENABAR I., M., LÓPEZ-ARRAIZA A., LIZARANZU M., AURREKOETXEA J. 2011. Comparison and analysis of non-destructive testing techniques suitable for delamination inspection in wind turbine blades Composites Part B: Engineering, 42.

AULD, B. A. 1990. Acoustic Fields and Waves in Solids, second edition.
BALASUBRAMANIAM, K. \& WHITNEY, S. C. 1996. Ultrasonic through-transmission characterization of thick fibre-reinforced composites. Ndt \& E International, 29, 225-236.

DAGGUMATI, S., VAN PAEPEGEM, W., DEGRIECK, J., XU, J., LOMOV, S. V. \& VERPOEST, I. 2010. Local damage in a 5-harness satin weave composite under static tension: Part II - Meso-FE modelling. Composites Science and Technology, 70, 1934-1941.

DECLERCQ, N. F., DEGRIECK, J. \& LEROY, O. 2004. On the influence of fatigue on ultrasonic polar scans of fiber reinforced composites. Ultrasonics, 42, 173-177.

DECLERCQ, N. F., DEGRIECK, J. \& LEROY, O. 2006 a. Simulations of harmonic and pulsed ultrasonic polar scans. Ndt \& E International, 39, 205-216.

DECLERCQ, N. F., DEGRIECK, J. \& LEROY, O. 2006 b. Ultrasonic polar scans: Numerical simulation on generally anisotropic media. Ultrasonics, 45, 32-39.

DEGRIECK, J., DECLERCQ, N. F. \& LEROY, O. 2003. Ultrasonic polar scans as a possible means of nondestructive testing and characterisation of composite plates. Insight, 45, 196-201.

I.A.VIKTOROV 1967. Rayleigh and Lamb waves, Plenum, New York.

NAYFEH, A. H. \& CHIMENTI, D. E. 1988. Ultrasonic wave reflection from liquid-coupled orthotropic plates with application to fibrous composites. Journal of Applied Mechanics-Transactions of the Asme, 55, 863-870.

ROSE, J. L. 1999. Ultrasonic Waves in Solid Media, Cambridge University Press.

VANDREUMEL, W. H. M. \& SPEIJER, J. L. 1981. Nondestructive composite laminate characterization by means of ultrasonic polar-scan. Materials Evaluation, 39, 922-925. 